\title{
GCU
}

Glasgow Caledonian

University

University for the Common Good

\section{Prototype gesture recognition interface for vehicular head-up display system}

Lagoo, Ramesh; Charissis, Vassilis; Chan, Warren; Khan, Soheeb; Harrison, David

Published in:

2018 IEEE International Conference on Consumer Electronics (ICCE)

DOI:

10.1109/ICCE.2018.8326146

Publication date:

2018

Document Version

Author accepted manuscript

Link to publication in ResearchOnline

Citation for published version (Harvard):

Lagoo, R, Charissis, V, Chan, W, Khan, S \& Harrison, D 2018, Prototype gesture recognition interface for vehicular head-up display system. in 2018 IEEE International Conference on Consumer Electronics (ICCE). IEEE, pp. 1-6, IEEE International Conference on Consumer Electronics , Las Vegas, United States, 12/01/18. https://doi.org/10.1109/ICCE.2018.8326146

\section{General rights}

Copyright and moral rights for the publications made accessible in the public portal are retained by the authors and/or other copyright owners and it is a condition of accessing publications that users recognise and abide by the legal requirements associated with these rights.

Take down policy

If you believe that this document breaches copyright please view our takedown policy at https://edshare.gcu.ac.uk/id/eprint/5179 for details of how to contact us. 


\title{
Prototype Gesture Recognition Interface for Vehicular Head-Up Display System
}

\author{
Ramesh Lagoo, Vassilis Charissis, Warren Chan, Soheeb Khan \& David Harrison*
}

\begin{abstract}
The proliferation of infotainment systems in the contemporary vehicular environment offers an abundance of information which is typically not distilled or prioritised according to the driver's requirements and driving conditions. Additionally, this requires from the driver to operate multiple interfaces, resulting in an overwhelming cognitive load and a higher probability of collision. Our work presents a new multimodal Head-Up Display interface that aims to enhance human responses during a potential collision whilst moderate the plethora of incoming information through a simplified and manageable system. The latter is fully interactive and operated with the use of an embedded gesture recognition system. The proposed system was evaluated by comparison to a traditional Head-Down Display interface system by twenty users, with the use of a high-fidelity driving simulator. The results were in favour of the proposed system which reduces the collision propensity by $\mathbf{9 0 \%}$ in a motorway environment.
\end{abstract}

Index Terms-Head Up Display, Displays, Driving Simulator, Traffic Flow, Collision Avoidance, Gesture Recognition, Infotainment System

\section{INTRODUCTION}

$\mathrm{M}$ IND-sharing notifications of an assortment of infotainment devices present inside the vehicle can absorb the driver's concentration on ineffective gazing at the dashboard dials, incoming mobile communications and navigation data in the case of Head-Down Displays (HDD), as well as on discerning the challenging road-traffic flow [1,2].

Therefore, in a potential abrupt braking of the lead vehicles the driver does not have the required time and situational awareness (figure 1) to proceed in a collision avoidance braking manoeuvre $[2,3]$.

Mr Ramesh Lagoo is $\mathrm{PhD}$ candidate at the Department of Computers Communication and Interactive systems, Glasgow Caledonian University, Glasgow, UK, (lagoo@gcu.ac.uk).

Professor Vassilis Charissis, is Director of the Virtual Reality and Simulation Laboratories (VRS Labs), Department of Computers Communication and Interactive systems, Glasgow Caledonian University, Glasgow, UK, (e-mail: vassilis.charissis@gcu.ac.uk).

Dr Warren Chan, is with the Department of Computers Communication and Interactive systems, Glasgow Caledonian University, Glasgow, UK, (e-mail: warren.chan@gcu.ac.uk).

Dr Soheeb Khan, is with the Department of Computers Communication and Interactive systems, Glasgow Caledonian University, Glasgow, UK, (e-mail: warren.chan@gcu.ac.uk).

Professor David K. Harrison is with the Mechanical and Electrical Engineering Department, Glasgow Caledonian University, Glasgow, UK (e-mail:dkh1@gcu.ac.uk).
In particular, numerous studies have explored the issues created by the use of mobile in-vehicle communication by the driver [4,5 \& 6]. An attempt to educate the users on this matter has been pursued through different government and manufacturers campaigns with limited results [7]. Similar issues have also been observed during the programming of navigation devices on-route. The stimulus overload in both cases can have detrimental results in imminent collision scenarios [2,3].

Based on our previous experience, a Head-Up Display (HUD) interface could offer a significant advantage in the provision of crucial information to the driver in the extensive vehicular windshield estate [1, 2 \& 3]. Concurrent HUD interface studies support further the use of HUD for improving driver's response times and collision avoidance capabilities [8, 9, 10 \& 11]. In this work, we have evolved our initial visual-only HUD interface to a multimodal HUD that could be fully interactive with the use of gesture recognition system. In turn, the paper describes succinctly the in-parallel development of a new high-fidelity driving simulator, which provided the testing ground for evaluation of the proposed system in contrast to typical Head-Down Display (HDD).

This work presents an innovative approach which combines the visual presentation of important information through a Head-Up Display system whilst enabling the driver to interact with the system in real-time through a novel Gesture Recognition System.

\section{Gesture ReCOGNITION CONTROLS}

The utilization of gestural control as compared to conventional controls offers the advantage of performing control tasks without affecting the visual and cognitive attention required for driving [12]. Notably, the gestures contain information and can be performed without taking eyes-off-the-road. Hence, they are ideal for developing specific in-vehicle interaction tasks that can minimise driver distraction. An additional benefit of these types of natural gesture is that they can be easily memorised, causing minimum cognitive driver workload, making them an attractive proposition for use with in-vehicle secondary controls. This has been documented by previous studies where participant drivers reported less distraction and had less error in 
performance when utilizing gestural controls as compared to haptic controls $[13,14]$.

Unlike speech-based controls, gestural controls are not affected by noisy conditions, dialects and accents that can be source of error for speech based controls.

Non-contact gestural acknowledgment additionally offers multiple valuable benefits. However, the most important benefit for the in-vehicle interface is the absence of physical contact with any form of screen or surface. As such the driver is not required to focus his/her attention to touch a particular point in the HDD that could perform the required task as presented in previous studies [15].

Additionally, the drivers of the aforementioned studies also favoured the gestural controls compared to conventional controls.

Acceptance models have indicated that gestural controls would be readily accepted by the drivers for controlling secondary tasks if these can be performed with one hand without distraction [16]. This supports the hypothesis that gestures are a rightful alternative for current paradigm of physical interaction by requiring minimum workload for operation, and less continuous moves. This could improve operation proficiency and consequently it could increase road safety [16]. The functionality of a gesture based system could however be affected if the gesture interfaces are not self-revealing, so the user has to know beforehand the specific set of gestures that the system understands. As such it becomes more onerous for the user to memorise the correct gestural command set as the number of gestures increase. This is an evident problem with some early adaptations of gesture recognition technology by some automotive manufacturers. In order to avoid this issue, our proposed gesture recognition interface is seamlessly coupled with a visual Head-Up Display interface as seen in Figure 1.

\section{PROPOSED SYSTEM}

In our prior work we have developed a prototype Head-Up Display (HUD) interface in order to improve driver's response times (RT) and situational awareness during driving under adverse weather conditions and low visibility [1, $2 \& 3]$. Due to the provided windshield estate and proximity to driver's field of view, a HUD solution was deemed essential for a different set of functionalities aiming to minimise driver distraction by controlling the multiple stimuli provided by in-vehicle infotainment devices.

In this first stage, the proposed interface was designed to set the working framework of visual interface that could maintain driver's attention on the road and regulate primarily the incoming communication and navigation requirements. The proposed system employs a sequence of interrupt strategies that prioritise the release of information to the driver depending on the speed of the vehicle, traffic flow and weather conditions. The prototype HUD interface is comprised of the visual and gesture recognition interface elements as illustrated in Figure 1 below.

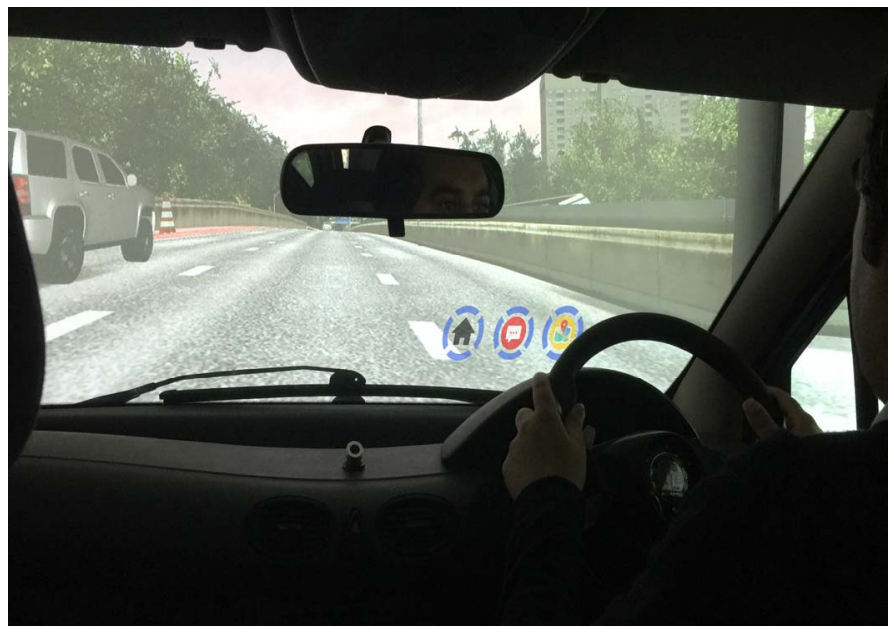

Figure 1: Screenshot of the actual VR Driving Simulator with enabled HUD interface.

\section{A. Visual Interface}

The visual interface has been developed through an iterative process of focus group testing and elimination of unnecessary information which might distract the driver. The interface design provided clear visual indications primarily for the regulation of mobile phone calls, text messaging and navigation information.

The initial system was designed with six icons namely; (a) Home, (b) Settings, (c) Internet (d) Text Message, (e) Phone Call, and (f) Map/Navigation. The first three icons served as generic and calibration functions whilst the remaining three aimed for some of the most time-consuming and attention-seeking actions.

The system was evaluated by the focus group and the initial user feedback suggested that although the visual information was functional, the interaction with the icons through a gesture recognition was burdened from the precision required to "air-click" the icons. In order to improve the interactivity, various methods have been implemented and tested such as dynamic alteration of icon size or different activation methods which did not resolve the selection issue.

In turn, the redesigned visual interface reduced to three functional icons each time, in order to limit the selection points and improve the system usability. The icons selected were (a) Home, (b) Map/Navigation and (c) Text Message as presented in Figure 2.

The Settings and Internet icons were considered obsolete for direct use in the HUD environment and were transferred to the HDD screen as both should be activated when the vehicle is 
immobile, and safely parked. Notably both actions were time consuming and their attention seeking properties rendered them unusable for direct manipulation through the HUD gesture recognition system. The Text Message icon entails an interface duality which either provides a subtle warning about incoming text message or a different standardised warning for a mobile phone call that has been blocked. The latter icon provides a simple metadata of the missed call which includes the name of caller, and time.

Notably, the system will be activated when the vehicle moves in high speeds (motorway environment), or in the dense urban environment. The system will also take into consideration the weather conditions and depending on the adversity it will block the call or transfer it to the vehicle speakers. As such the three main icons considered usable for this type of HUD interface were the Home icon, Text Message icon and the Navigation icon.

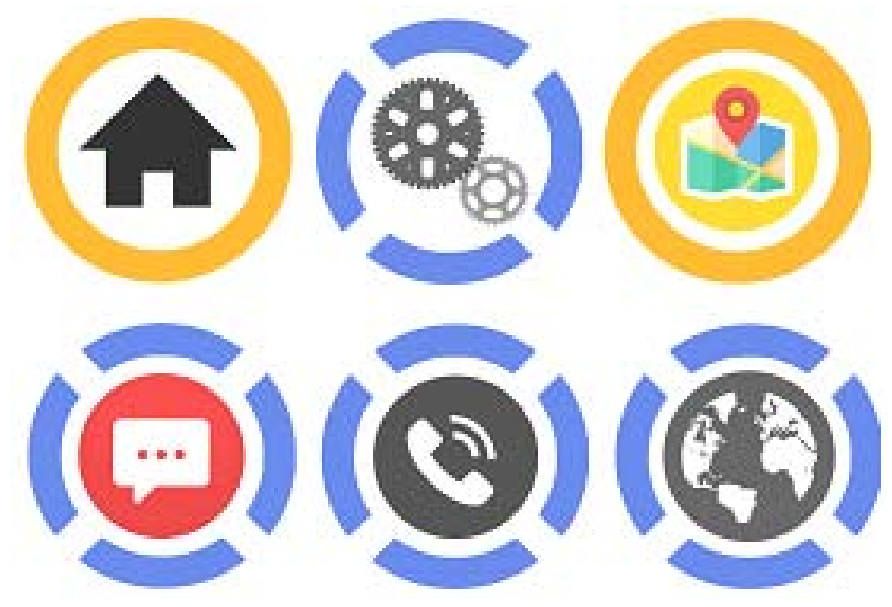

Figure 2. Initial HUD interface entailing all six icons namely: (a) Home icon, (b) Settings Icon (Idle state), (c) Navigation/Map Icon (Hover state), (d) Text Message Icon (Warning State), (e) Phone Call Icon (Idle State) \& (f) Internet Icon (Idle State)

\section{B. Gesture Recognition Interface}

Adhering to the aforementioned iterations the three-icon set-up required a feasible gesture vocabulary that was memorable and readily usable by the drivers [17].

The clarity of each gesture and time of completion was imperative to be performed within specific spatial and time limits [18]. The sensor was positioned on the main hub above and behind the steering wheel in proximity to the driver's hand. This position presented the opportunity for the use of more feasible gestures and combination of dynamic and technology based gestures.

The dynamic and spatiotemporal gestures were reduced to a straightforward movement towards the icon, imitating the clicking process of an actual button. This was evident after initial trials which utilised typical dynamic gestures such as next, stop, quit and start presented in Figure 3 (a).

Interestingly the particular moves were deemed unusable as they required approximately 2 seconds for the completion of each move and more importantly to lift the hand completely from the steering wheel. The latter defined the purpose of a gesture interface, which aimed to avoid the activation of buttons or levers away from the steering wheel area.

The technology based gestures, utilised the selection of icons through the use of a different number of fingers each time between the index, middle and ring fingers as presented in Figure 3 (b). This combinatory approach resulted in a fast and efficient selection of buttons minimising the false selections and the duration of the moves. However, during the preliminary trials, it became apparent that the use of multiple finger gestures was challenging and time consuming during the driving process. As such we simplified even further the interface and utilised only one finger (pointer) selection. The latter system alteration increased the speed of movements and the response of the HUD interface.
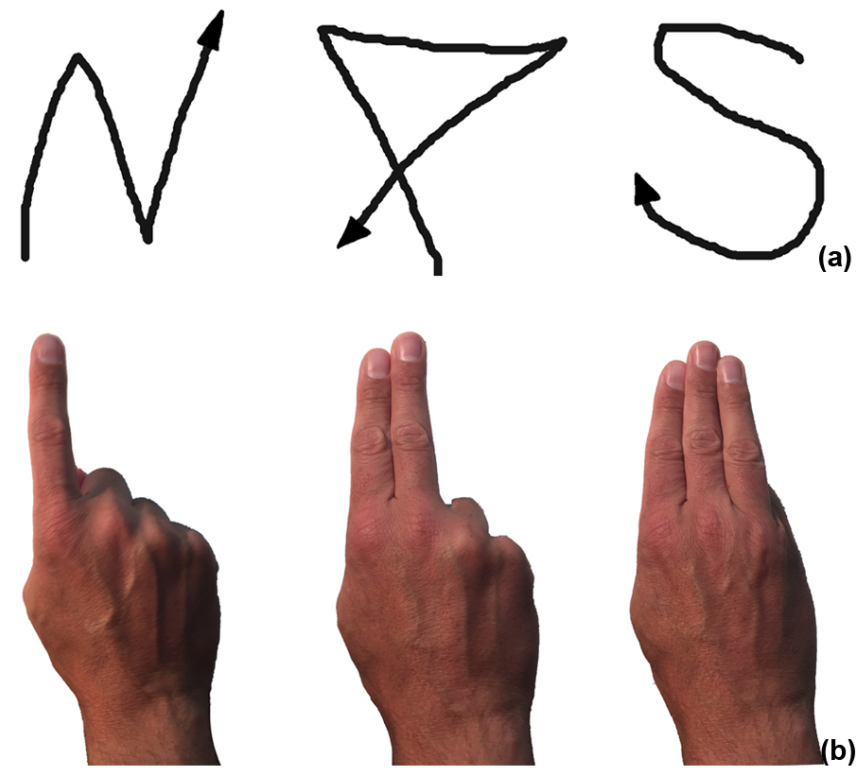

Figure 3. (a) Dynamic Gestures (Next, Quit, Start; (b) Technology Gestures (Use of 1-3 fingers)

\section{Methodology}

Consequently, the complete proposed HMI system has been evaluated in our 4th generation Virtual Reality Driving Simulator developed explicitly to measure drivers' performance with the proposed HUD interface and compare its effectiveness to traditional instrumentation techniques.

The evaluation process employed both Qualitative \& Quantitative methodologies. The qualitative evaluation entailed a pre-test questionnaire aiming to gather information regarding driving experience, mobile and computer technologies, as well as driving habits. By the completion of the driving simulation 
experiments, the participants were given a post-test questionnaire to attain knowledge regarding the usability feedback of the proposed system.

The analysis of the results gathered from this pilot study ranged from neutral to positive appraisal of the system. The results also pointed towards required improvements and investigations required to improve the system.

The quantitative part of the evaluation was measured through the number of collisions that occurred per trial, the actual response times (RT) and headway (HW) benefits derived through its usage and subsequently the real impact in the decrease of accident propensity $[3,4]$.

In turn a modified Technology Acceptance Model (TAM) is used for the qualitative evaluation process of the users' subjective feedback with focused interest in three antecedents, namely; perceived ease of use, perceived ease of usefulness and perceived enjoyment. However, in this paper we present the preliminary results from the users' collisions with and without the use of the proposed HUD interface.

\section{Simulation REQuiREMENTS}

\section{A. Evaluation Scenarios}

The proposed HUD system was evaluated through a comparative study which contrasted existing systems and the proposed interface in two typical driver distraction scenarios presented below.

The simulation scenario based on a compilation of potential accident scenarios provided by Strathclyde Police department and used with minor variations of our previous simulation experiments [1, 2 \& 3].

This was deemed necessary in order to maintain the consistency between the different trials whilst different interface design and attributes are evaluated. The scenario scheme aims to distract the driver with the use of random text messages in demanding, high speed, traffic situation.

The system measures predominantly the human responses through the Response Time required for the safe braking in an imminent collision situation in a motorway environment.

\section{B. Participants}

The evaluation was performed by twenty users ( 7 female, 13 male) which held a valid driving licence and they were aged between 20 and 55. For maintaining the validity of the experiment, the Artificial Intelligence on the simulation vehicles was programmed to follow the overall manoeuvring, speed and distances described in the British Highway Code.

\section{Driving Simulator}

A driving simulator was deemed an ideal system for the system evaluation, as it is offering a controlled environment to experiment safely $[19,20]$. As such, we have developed a new Virtual Reality (VR) driving simulator, designed explicitly for the testing of vehicular interfaces. The new simulator utilises a full-scale Mercedes A-Class vehicle, positioned in a CAVE room. The latter offers a full enclosure and surround projection which increases the immersion sensation. Additionally, the new simulator improves on graphics and physics fidelity over previous works $[2,3]$. The simulator interior provides multiple touchscreens for the simulation of HDD, fully functional dashboard instrumentation and Leap-motion sensors positioned on the top of the dashboard hub for the facilitation of the gesture recognition. Surround audio and vibrating devices positioned on the vehicle floor enhance further the driving experience. During each user trial, the simulator recorded the response time in the imminent collision situations and the actual number of collisions occurred. Additionally, the system logged the driver's speed, lane position, distance from the lead vehicle and simulation elapsed time per second.

\section{Evaluation Results}

The evaluation process aimed to identify the benefits and potential issues of both HDD and HUD systems. The simulation scenario utilises a motorway environment with light traffic flow. During the simulation, the driver receives text messages in random intervals presented on a tablet device positioned in the middle of the vehicle dashboard. The requested task is to open and read the message whilst driving.

The collision results with and without the HUD interface are presented below in Figure 3. Evidently the use of the HUD resulted in a drastic drop in the number of cases where a collision occurred and a sharp increase in the number of uneventful runs.

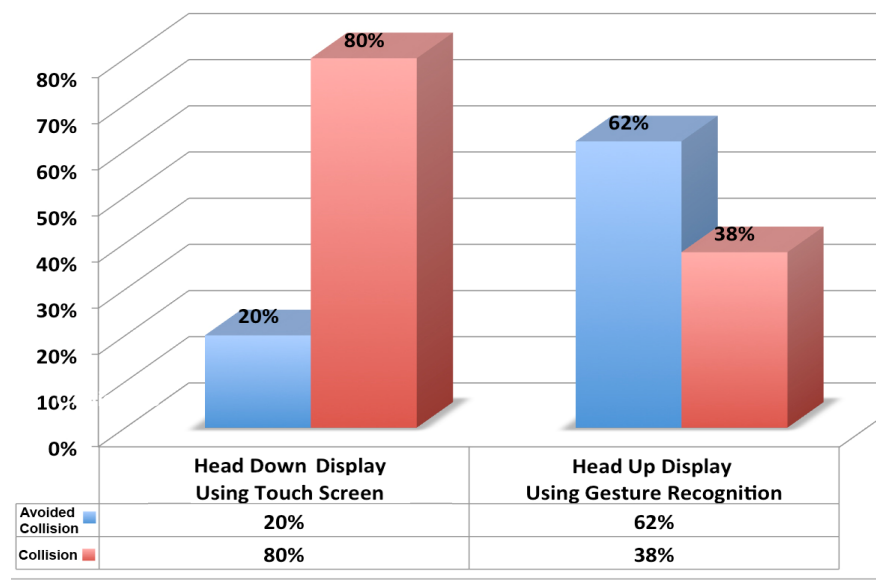

Figure 3. Number of collisions recorded with and without the HUD interface 


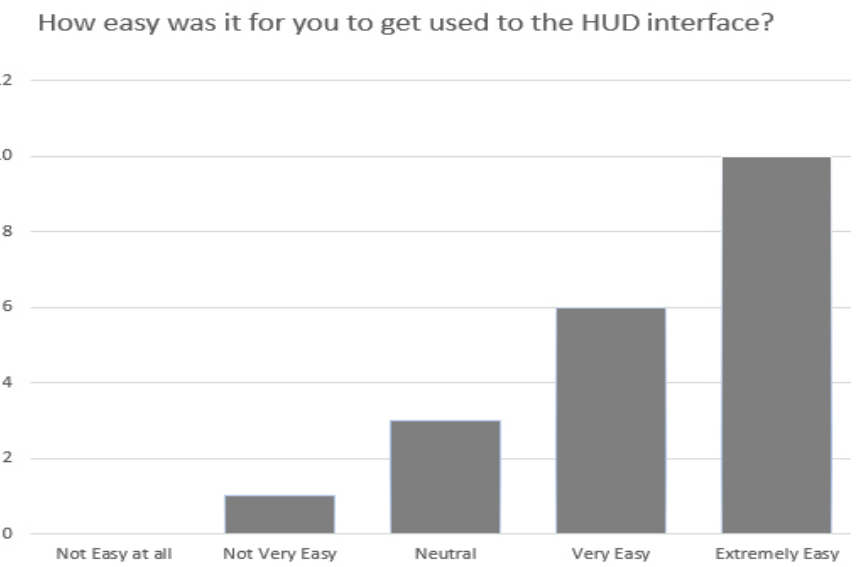

Figure 4. User Experience results regarding the ease of use for the HUD interface

The collisions dropped by $42 \%$ with the use of the HUD and Gesture Recognition interface. Furthermore, the User Experience (UX) analysis highlighted that $80 \%$ of the users found it very or extremely easy to use the particular gesture recognition HUD interface as presented above in Figure 4.
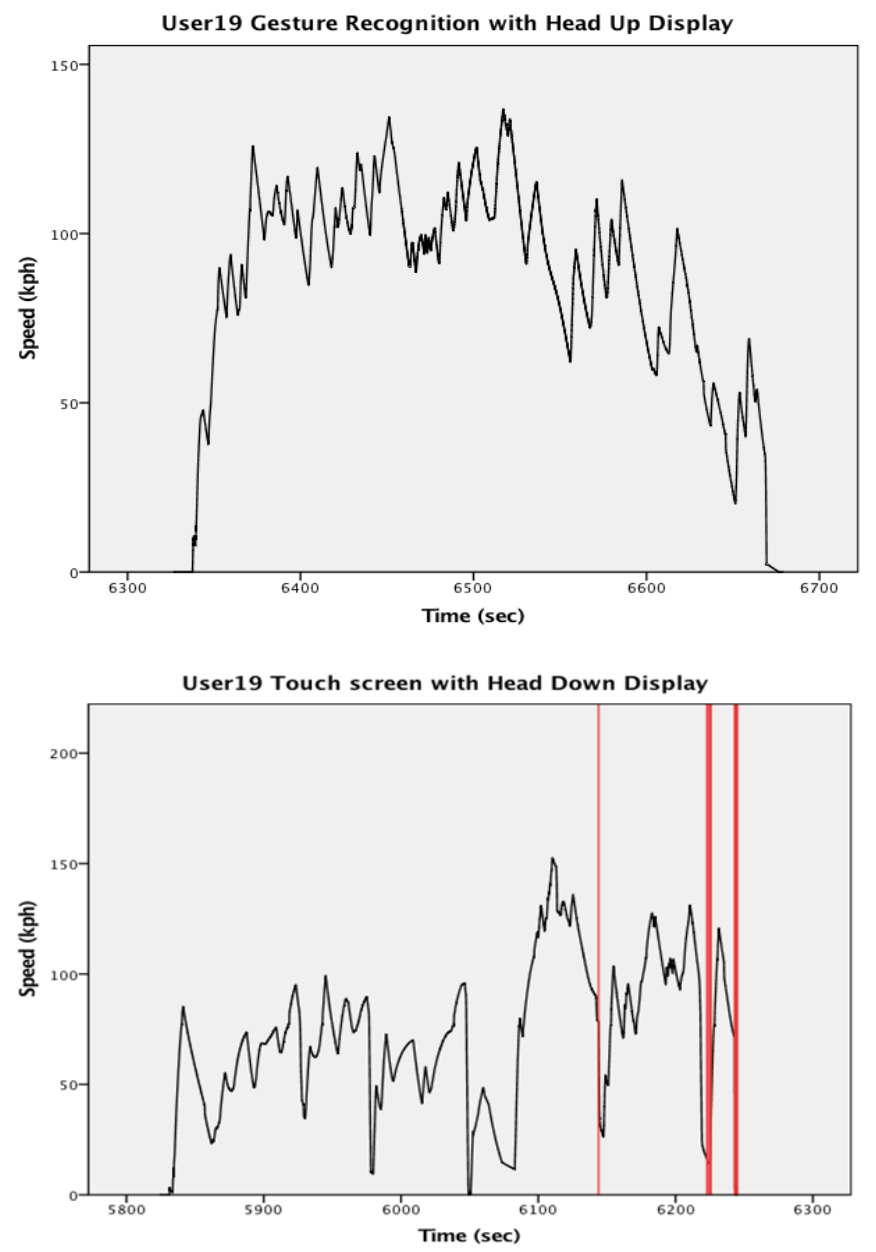

Figure 5. Indicative sample of user's driving pattern with and without the proposed HUD/Gesture Recognition interface.

Finally, the analysis has presented a notably relaxed pattern in the driving responses with a large reduction in abrupt braking or lane changing tendencies as presented in one of the indicative user trials in Figure 5. The particular driver presented a controlled response with the use of the HUD and avoided the pre-determined accident spots.

In contrast, using the HDD, the driver was temporarily distracted with the incoming messages and the navigation data which resulted in ten collisions throughout the experiment. The last nine collisions occurred when the driver was preoccupied with the HDD information and selection menus. Momentarily the driver attempts to avoid the imminent collision by braking abruptly and steering the vehicle. Unfortunately, this typical response entangled the driver in a multiple collision that in real-life would have resulted to a fatal pile-up of vehicles. Notably the aftermath of the simulation presented ten collisions with six vehicles and four different sections of the road barriers.

\section{CONCLUSIONS}

This paper presented the design and implementation challenges of our prototype multimodal HUD interface that aims to reduce the number of attention seeking infotainment interfaces and improve the response time and situational awareness of the driver.

For the evaluation of the system, we have developed a high fidelity full-scale driving simulator that replicated realistically two driving scenarios of high probability collision. The system has been preliminarily evaluated by twenty users with promising results, demonstrating $42 \%$ improvement in collision avoidance as the users maintained the eye gaze on the road and their hands on the steering wheel at all times.

Out tentative plan of future work entails the optimisation of the gesture recognition system and the incorporation of additional visual icons so they can also be engaged by the co-driver and remotely be transferred within the vehicle environment. Furthermore, we are planning to continue the user evaluation experiments in order to accumulate more conclusive results regarding the system efficiency and usability.

\section{REFERENCES}

[1] V. Charissis, W. Chan, S. Khan, R. Lagoo, "Improving Human Responses with the use of prototype HUD interface", ACM SIGGRAPH Asia 2015, Kobe, Japan, 2015.

[2] V. Charissis, S. Papanastasiou, W. Chan, and E. Peytchev, "Evolution of a full-windshield HUD designed for current VANET communication standards", IEEE Intelligent Transportation Systems International Conference (IEEE ITS), The Hague, Netherlands, pp. 1637-1643, DOI: 10.1109/ITSC.2013.6728464, 2013.

[3] V. Charissis, and S. Papanastasiou, S.,"Human-Machine Collaboration Through Vehicle Head-Up Display Interface", in International Journal of Cognition, Technology and Work, P. C. Cacciabue and E. Hollangel (eds.) Springer London Ltd Volume 12, Number 1, pp 41-50, DOI: 10.1007/s10111-008-0117, 2010.

[4] N. Reed, \& A. Parkes, "The Effects of Text Messaging on Driver Behaviour A simulator study", Transport Research Laboratory, 2008. 
[5] F. A., Dews, and D. L., Stayer, "Cellular Phones and Driver Distraction". In M. A. Regan, J. D. Lee, \& K. L. Young, Driver Distraction Theory, Effects and Mitigation, pp. 169-190, CRC Press, 2009.

[6] S.M. Simons, A. Hicks, J. K. Caird, Safety-critical event risk associated with cell phone tasks as measured in naturalistic driving studies: A systematic review and meta-analysis, Accident Analysis and Prevention Journal, Elsevier, Vol 87, pp 161-169, 2016.

[7] A. Rohl, S. Eriksson, and D Metcalf, "Evaluating the Effectiveness of a Front Windshield Sticker Reminder in Reducing Texting while Driving in Young Adults", Cureus, 8(7), e691, 2016.

[8] H. Okumura \& K. Shinohara, "Human Attention and fatigue for AR Head-Up Displays" 2016 IEEE International Symposium on Mixed and Augmented Reality (ISMAR-Adjunct), Merida, Mexico, pp. 306-309, 2016.

[9] H. Okumura, T. Sasaki, A. Hotta, and K. Shinohara, "Monocular hyperrealistic virtual and augmented reality display" 2014 IEEE Fourth International Conference on Consumer Electronics (ICCE-Berlin), Berlin, Germany, pp.19-23, 2014

[10] J. L. Gabbard, M. G. Fitch, K. Hyungil, "Behind the Glass: Driver Challenges and Opportunities for AR Automotive Applications", Proceedings of the IEEE, Vol. 102:2, pp.124-136, DOI: 10.1109/JPROC.2013.2294642, 2014.

[11] K. Hyungil, W Xuefang, J.L. Gabbard, N.F. Polys, "Exploring head-up augmented reality interfaces for crash warning systems", 5th International Conference on Automotive User Interfaces and Interactive Vehicular Applications, Automotive UI '13, pp. 224-227, ACM, 2013.

[12] M. Koelle, "Freehand Gestures for Interactions in Automobiles: a technology-based View”. In Ubiquitous Computing, 2011.

[13] M. Zobl, M. Geiger, K. Bengler, and M. Lang, M., "A usability study on hand gesture controlled operation of in-car devices." Abridged Proceedings, HCI, pp.5-10, 2003.

[14] M. Alpern, \& K. Minardo, "Developing a car gesture interface for use as a secondary task", In ACM CHI'03 extended abstracts on Human factors in computing systems, pp. 932-933, 2003.

[15] C.A. Pickering, K.J. Burnham, and M.J. Richardson, "A review of automotive human machine interface technologies and techniques to reduce driver distraction", In 2nd IET Conf. on System Safety, pp. 223-228, 2007.

[16] B. Reimer, B. Mehler, J. Dobres, and J.F. Coughlin, "The effects of a production level "voice-command" interface on driver behavior: reported workload, physiology, visual attention, and driving performance", MIT Age-Lab Technical Report, (No. 2013-17A), 2013.

[17] K. W. Gish, \& L. Staplin, "Human factors aspects of using head up displays in automobiles: A review of the literature", (Report No. DOT HS 808 320). Washington, DC: National Highway Traffic Safety Administration, 1995.

[18] M. Nielsen, T. B. Moeslund, M. Storring and E. Granum, "HCI Beyond the GUI, Design for Haptic, Speech, Olfactory, and Other Non-traditional Interfaces", Kortum, P., (Ed), Elsevier, Morgan Kaufmann Publishers, pp.75-103, 2008.

[19] G. Underwood, D. Crundall, and P. Chapman, "Driving simulator validation with hazard perception", Transportation Research Part F: Traffic Psychology and Behaviour, vol. 14, no. 6, pp. 435 - 446, Special Issue: Driving Simulation in Traffic Psychology, 2011.

[20] G. Weinberg, B. Harsham, and Z. Medenica, "Evaluating the usability of a head-up display for selection from choice lists in cars", 3rd International Conference on Automotive User Interfaces and Interactive Vehicular Applications, Automotive UI '11, pp. 39-46, ACM, 2011. 